\title{
Revision Analysenliste - quo vadis?
}

\author{
Ernst Gähler ${ }^{a}$, Beat Dubs ${ }^{b}$ \\ a Mitglied des Zentralvorstandes \\ der FMH \\ b Tarifverantwortlicher KHM
}

\begin{abstract}
Ausgangslage
Das BAG beschliesst 2006 eine Revision der Analysenliste auf dem Gebiet der Nomenklatur und der Preisbildung. Am 19. Oktober 2006 nimmt eine Delegation der FMH (vertreten durch SGAM, SGIM, SFSM, SULM und ZV FMH) an einem Hearing teil. Das BAG will in Zukunft mit einem betriebswirtschaftlich gerechneten Tarif nur noch einen Taxpunktwert (TPW) für alle Laborleistungen in der Analysenliste (AL) festhalten. Die Analysen sollen unabhängig davon, wo sie erbracht werden, gleich taxiert werden. Differenzierte TPW für die speziellen Labortypen (Praxislabor, Privatlabor, Spitallabor usw.) sind nicht mehr möglich.

Die FMH-Delegation bringt an diesem Hearing folgende Punkte ein:

- mögliche «Verluste» für das Praxislabor sollen durch sogenannte Vorhaltekosten abgedeckt werden;

- diese enthalten eine Taxe im Präsenzlabor, die die Abgeltung der intellektuellen Leistung und einen Gewinn ermöglichen;

- damit sollen auch die ungleich höheren Aufwendungen im Präsenzlabor der Praxis ausglichen werden.
\end{abstract}

\section{So ging es weiter}

Das BAG setzt zur Vorbereitung des Projekts als Projektgruppe die ZMT/MTK (der Unfallversicherer) ein, die im Frühjahr 2007 ausgewechselt wird. Lange Zeit herrscht Funkstille vom BAG betreffend neue Projektgruppe und weiteres Vorgehen.

Im Sommer 2007 wird ein breitabgestütztes Positionspapier (SULM, santésuisse, H+, FAHM,
FMH, SLSA, GDK, SVDI, SAMW) zur Revision AL zuhanden des BAG erstellt, das die FMH mitunterzeichnet hat. Für uns wichtigster Punkt in diesem Konsenspapier ist das klare Bekenntnis der anderen Player zur Erhaltung des Praxislabors und zur Abgeltung der «Nachteile» des Praxislabors durch eine AIP (Administrativ- und Infrastrukturpauschale).

Das BAG informiert an der Sitzung der Analysenlistenkommission vom 20. August 2007, dass die Änderung des TPW und der Tarifstruktur per 1. Juli 2008 erfolgt.

\section{Projektgruppe Labor (AL/QUALAB)}

Aus dem Büro Tarife der FMH (FMCH, KHM, KKA, SFSM, SGGP, Ressort Tarife) wird eine Projektgruppe Labor gebildet, die aus drei Vertretern des KHM, zwei Vertretern der KKA, zwei Experten und dem Ressortverantwortlichen der FMH besteht. Die Projektgruppe nimmt die Arbeit auf. Wichtig ist es, eigenes Zahlenmaterial zu sichten und zu generieren, damit die Verhandlungen mit dem BAG optimal vorbereitet werden können. $\mathrm{Ob}$ es eine lineare Absenkung des TPW oder eine differenzierte Anpassung einzelner Analysen geben wird, ist noch nicht bekannt.

Unser Ziel muss der Erhalt des Praxislabors unter folgenden Gesichtspunkten sein:

- Schaffen optimaler Berechnungsgrundlagen für die AIP (Administrativ- und Infrastrukturpauschale);

- die Abgeltung der AIP muss im Minimum kostenneutral erfolgen;

- regelmässige Information über den Verlauf. 